\title{
Antimicrobial resistance in fecal Escherichia coli and Salmonella enterica isolates: a two- year prospective study of small poultry flocks in Ontario, Canada
}

\author{
Csaba Varga ${ }^{1 *}$ (D) Michele T. Guerin ${ }^{2}$, Marina L. Brash ${ }^{3}$, Durda Slavic ${ }^{3}$, Patrick Boerlin ${ }^{4}$ and Leonardo Susta ${ }^{4}$
}

\begin{abstract}
Background: Although keeping small poultry flocks is increasingly popular in Ontario, information on the antimicrobial susceptibility of enteric bacteria of such flocks is lacking. The current study was conducted on small poultry flocks in Ontario between October 2015 and September 2017, and samples were submitted on a voluntary basis to Ontario's Animal Health Laboratory. From each submission, a pooled cecal sample was obtained from all the birds of the same species from the same flock and tested for the presence of two common enteric pathogens, E. coli and Salmonella. Three different isolates from each E. coli-positive sample and one isolate from each Salmonellapositive sample were selected and tested for susceptibility to 14 antimicrobials using a broth microdilution technique.

Results: A total of 433 fecal E. coli isolates (358 chicken, 27 turkey, 24 duck, and 24 game bird) and 5 Salmonella isolates (3 chicken, 1 turkey, and 1 duck) were recovered. One hundred and sixty-seven chicken, 5 turkey, 14 duck, and 15 game bird E. coli isolates were pan-susceptible. For E. coli, a moderate to high proportion of isolates were resistant to tetracycline (43\% chicken, $81 \%$ turkey, $42 \%$ duck, and 38\% game bird isolates), streptomycin (29\% chicken, 37\% turkey, and 33\% game bird isolates), sulfonamides (17\% chicken, 37\% turkey, and 21\% duck isolates), and ampicillin ( $16 \%$ chicken and $41 \%$ turkey isolates). Multidrug resistance was found in 37\% of turkey, $20 \%$ of chicken, $13 \%$ of duck, and $8 \%$ of game bird E. coli isolates. Salmonella isolates were most frequently resistant to streptomycin, tetracycline, and sulfonamides. Resistance to cephalosporins, carbapenems, macrolides, and quinolones was infrequent in both E. coli and Salmonella isolates. Cluster and correlation analyses identified streptomycin-tetracyclinesulfisoxazole-trimethoprim-sulfamethoxazole as the most common resistance pattern in chicken E. coli isolates. Turkey E. coli isolates compared to all the other poultry species had higher odds of resistance to tetracycline and ampicillin, and a higher multidrug resistance rate.
\end{abstract}

Conclusions: Escherichia coli isolates were frequently resistant to antimicrobials commonly used to treat poultry bacterial infections, which highlights the necessity of judicious antimicrobial use to limit the emergence of multidrug resistant bacteria.

Keywords: Antimicrobial susceptibility, Multidrug resistance, Chicken, Turkey, Poultry, Backyard flock, Antibiotic, Surveillance, Longitudinal study

\footnotetext{
* Correspondence: cvarga@uoguelph.ca

${ }^{1}$ Department of Pathobiology, College of Veterinary Medicine, University of

Illinois at Urbana-Champaign, Urbana, Illinois 61802, USA

Full list of author information is available at the end of the article
}

(c) The Author(s). 2019 Open Access This article is distributed under the terms of the Creative Commons Attribution 4.0 International License (http://creativecommons.org/licenses/by/4.0/), which permits unrestricted use, distribution, and reproduction in any medium, provided you give appropriate credit to the original author(s) and the source, provide a link to the Creative Commons license, and indicate if changes were made. The Creative Commons Public Domain Dedication waiver (http://creativecommons.org/publicdomain/zero/1.0/) applies to the data made available in this article, unless otherwise stated. 


\section{Background}

Non-commercial poultry flocks (denoted as "small flocks") are increasingly popular in urban, suburban, and rural areas in North America [1-3]. Small flocks can pose a health risk to their owners by exposing them to zoonotic pathogens [4-7] through consumption of contaminated meat or eggs $[8,9]$, or direct contact with infected birds [10] or their environment [11]. Antimicrobial resistance (AMR) in zoonotic pathogens adds to this risk [12-15] because infections with antimicrobial resistant bacteria are more difficult to treat, and result in higher morbidity and mortality [16, 17]. Inappropriate antimicrobial use has been shown to be one of the main causes for the development of AMR in commensal and pathogenic bacteria of poultry [17, 18]. Exposure to an individual antimicrobial may cause the bacteria to develop resistance to multiple antimicrobials if resistance genes are located on mobile genetic elements [19]. Furthermore, these acquired resistance determinants can persist even after the antibiotic selection pressure ends [12].

Health Canada categorizes antimicrobials based on their importance in human medicine: I - very high importance; II - high importance; III - medium importance; and IV - low importance [20]. The classification system considers the antimicrobial's indication (e.g., preferred choice for treatment of serious human infections) and availability of replacements (e.g., limited substitutes available) [20]. Under an amended Canadian regulation that came into effect on December 1, 2018, all medically important antimicrobials (Categories I, II, and III) used in food animals require a veterinary prescription in order to help limit the development and spread of AMR [21].

In Canada, AMR of Escherichia coli and Salmonella isolates obtained from commercial broiler chicken and turkey flocks is monitored by the Canadian Integrated Program for Antimicrobial Resistance Surveillance [22]. Emergence of resistance to antimicrobials commonly used to treat bacterial infections in commercial poultry flocks in Ontario is well documented [22-24]. In contrast, only one study [25], which was conducted in provincially inspected slaughter plants, has documented resistance to antimicrobials in small flocks in Ontario. Thus, the objectives of this study were to evaluate AMR patterns of fecal E. coli and Salmonella enterica isolates of chickens, turkeys, waterfowl, and game birds from Ontario small flocks submitted for laboratory diagnostic testing because of morbidity or mortality, and to determine differences in AMR patterns among different poultry species.

\section{Results}

Description of submissions

Over the 2-year period, the Animal Health Laboratory received 160 small flock submissions, with a median of 1 bird per submission (range $=1-5$ ), from flocks ranging in size from 1 to 299 birds (median 25) and birds ranging in age from 6 days to 7 years (median 7 months). The majority of submissions were chickens (84\%, 134 submissions), although a few turkey (10), duck (8), and game bird (8) submissions were also received [26].

\section{Antimicrobial resistance of Salmonella isolates}

Of 159 submissions tested for Salmonella spp. (a sample from one chicken submission was not available), a total of 5 isolates were recovered ( 5 pooled samples, 1 isolate recovered from each pooled sample). Serotypes included $S$. Anatum, S. Indiana, and S. Ouakam (3 chicken pooled samples), $S$. Uganda (1 turkey pooled sample), and $S$. Montevideo (1 duck pooled sample) [26]. Three Salmonella isolates were pan-susceptible (1S. Indiana, 1 $S$. Montevideo, and $1 S$. Uganda). The $S$. Anatum isolate was resistant to streptomycin, and the $S$. Ouakam isolate was multidrug resistant (streptomycin-sulfisoxazole-trimethoprim-sulfamethoxazole-tetracycline).

\section{Antimicrobial resistance of $E$. coli isolates}

Of 159 submissions tested for fecal E. coli, a total of 433 isolates were recovered: 358 from chicken submissions (120 pooled samples; 3 isolates recovered from 119 pooled samples and 1 isolate recovered from 1 pooled sample); 27 from turkey submissions (9 pooled samples, 3 isolates recovered from each pooled sample); 24 from duck submissions ( 8 pooled samples, 3 isolates recovered from each pooled sample); and 24 from game bird submissions ( 8 pooled samples, 3 isolates recovered from each pooled sample). Of these, $46.65 \%$ of the chicken (167/358), $18.52 \%$ of the turkey (5/27), $58.33 \%$ of the duck (14/24), and $62.50 \%$ of the game bird (15/24) isolates were pan-susceptible.

In the chicken $E$. coli isolates, there was a high frequency of resistance ( $\geq 40 \%$ of isolates) to tetracycline, a moderate frequency of resistance (15-39\% of isolates) to streptomycin, sulfisoxazole, and ampicillin, and a low frequency of resistance (5-14\% of isolates) to trimethoprimsulfamethoxazole and gentamicin (Table 1). All of the other antimicrobials tested had a very low frequency of resistance $(<5 \%)$. In the turkey $E$. coli isolates, there was a high frequency of resistance to tetracycline and ampicillin, and a moderate frequency of resistance to streptomycin and sulfisoxazole. In the duck E. coli isolates, there was a high frequency of resistance to tetracycline, and a moderate frequency of resistance to sulfisoxazole and trimethoprimsulfamethoxazole. In the game bird $E$. coli isolates, there was a moderate frequency of resistance to tetracycline and streptomycin.

In the chicken $E$. coli isolates, the most common AMR patterns were ampicillin-streptomycin-tetracycline (22 isolates, 6.15\%) and streptomycin-tetracycline (19 isolates, 
Table 1 Percentage of fecal Escherichia coli isolates from Ontario small poultry flocks that were resistant to 14 selected antimicrobials, as determined by a broth microdilution technique, by poultry species

\begin{tabular}{|c|c|c|c|c|c|}
\hline $\begin{array}{l}\text { Antimicrobial } \\
\text { class }\end{array}$ & Antimicrobial $^{\mathrm{A}}$ & $\begin{array}{l}\text { Chicken }(\mathrm{N}=358) \\
\mathrm{n}(\%)^{\mathrm{B}}[\mathrm{Cl}]^{\mathrm{C}}\end{array}$ & $\begin{array}{l}\text { Turkey }(\mathrm{N}=27) \\
\mathrm{n}(\%)[\mathrm{Cl}]\end{array}$ & $\begin{array}{l}\text { Duck }(N=24) \\
\text { n (\%) }[\mathrm{Cl}]\end{array}$ & $\begin{array}{l}\text { Game birds }(N=24) \\
\text { n (\%) }[\mathrm{Cl}]\end{array}$ \\
\hline \multirow[t]{2}{*}{ Aminoglycosides } & GEN & $23(6.42)$ [4.12-9.48] & 1 (3.70) [0.09-18.97] & 0 & 0 \\
\hline & STR & 105 (29.33) [24.66-34.34] & $10(37.04)$ [19.40-57.63] & 2 (8.33) [1.03-27.00] & 8 (33.33) [15.63-55.32] \\
\hline \multirow[t]{5}{*}{$\beta$-Lactams } & AMP & $57(15.92)$ [12.29-20.13] & $11(40.74)$ [22.39-61.20] & 1 (4.17) [0.11-21.12] & 1 (4.17) [0.11-21.12] \\
\hline & AMC & 8 (2.23) [0.97-4.36] & 2 (7.41) [0.91-24.29] & 0 & 0 \\
\hline & $\mathrm{CRO}$ & $4(1.12)$ [0.31-2.84] & $2(7.41)$ [0.91-24.29] & 0 & 0 \\
\hline & FOX & 5 (1.40) [0.46-3.23] & 1 (3.70) [0.09-18.97] & 0 & 0 \\
\hline & MER & 0 & 0 & 0 & 0 \\
\hline \multirow[t]{2}{*}{ Folate inhibitors } & SSS & 61 (17.04) [13.29-21.34] & 10 (37.04) [19.40-57.63] & 5 (20.83) [7.13-42.15] & 2 (8.33) [1.03-27.00] \\
\hline & STX & $30(8.38)$ [5.73-11.75] & 3 (11.11) [2.35-29.16] & 4 (16.67) [4.74-37.38] & 0 \\
\hline Macrolides & AZM & $5(1.40)[0.46-3.23]$ & 0 & 2 (8.33) [1.03-27.00] & 0 \\
\hline Phenicols & $\mathrm{CHL}$ & $17(4.75)$ [2.79-7.49] & 3 (11.11) [2.35-29.16] & 1 (4.17) [0.11-21.12] & 0 \\
\hline \multirow[t]{2}{*}{ Quinolones } & CIP & $1(0.28)$ [0.007-1.55] & $3(11.11)$ [2.35-29.16] & $1(4.17)$ [0.11-21.12] & 0 \\
\hline & NAL & 8 (2.23) [0.97-4.36] & 3 (11.11) [2.35-29.16] & 1 (4.17) [0.11-21.12] & 0 \\
\hline Tetracyclines & TET & 155 (43.30) [38.10-48.61] & $22(81.48)$ [61.92-93.70] & $10(41.67)$ [22.11-63.36] & 9 (37.50) [18.80-59.41] \\
\hline
\end{tabular}

${ }^{A}$ GEN gentamicin, STR streptomycin, AMP ampicillin, AMC amoxicillin-clavulanic acid, CRO ceftriaxone, FOX cefoxitin, MER meropenem, SSS sulfisoxazole, STX trimethoprim-sulfamethoxazole, AZM azithromycin, $C H L$ chloramphenicol, CIP ciprofloxacin, NAL nalidixic acid, TET tetracycline ${ }^{B}$ Number and percentage of isolates resistant to the antimicrobial

${ }^{\mathrm{C}} \mathrm{Cl}=$ Exact binomial $95 \%$ confidence interval for the percentage of isolates resistant to the antimicrobial

$5.31 \%$ ) (Table 2). The latter was also common in the game bird $E$. coli isolates.

Multidrug resistance was detected in $19.55 \%(95 \% \mathrm{CI}=$ 15.57-24.05) of the chicken, $37.04 \%$ (95\% CI $=19.40-$ $57.63)$ of the turkey, $12.50 \%(95 \% \mathrm{CI}=2.66-32.36)$ of the duck, and $8.33 \%(95 \% \mathrm{CI}=1.03-27.00)$ of the game bird E. coli isolates.

A high $(\geq 40 \%)$ proportion of $E$. coli-positive samples were resistant to tetracycline $(62.50 \%$ of the chicken, $100 \%$ of the turkey, $50 \%$ of the duck, and $57.14 \%$ of the game bird samples), streptomycin $(42.50 \%$ of the chicken, $55.56 \%$ of the turkey, and $42.86 \%$ of the game bird samples), ampicillin (55.56\% of the turkey samples), and sulfisoxazole (55.56\% of the turkey samples) (Fig. 1).

Single-linkage clustering dendrograms with Jaccard distances for E. coli resistance are presented in Fig. 2. A relatively high proportion (i.e., a cluster) of the chicken $E$. coli isolates were resistant to streptomycin, tetracycline, sulfisoxazole, and trimethoprim-sulfamethoxazole; a second cluster of chicken $E$. coli isolates was resistant to cefoxitin and ceftriaxone. Other notable clusters included resistance to streptomycin, sulfisoxazole, ampicillin, and tetracycline (turkey E. coli isolates), amoxicillin-clavulanic acid, cefoxitin, and ceftriaxone (turkey E. coli isolates), sulfisoxazole and trimethoprim-sulfamethoxazole (duck $E$. coli isolates), and streptomycin and tetracycline (game bird E. coli isolates). The turkey, duck, and game bird $E$. coli isolates were pan-susceptible to several antimicrobials.
The strongest, statistically significant pairwise correlations with respect to resistance of $E$. coli at the isolatelevel (chicken isolates only) were between cefoxitin and ceftriaxone $(\rho=0.67)$, sulfisoxazole and trimethoprimsulfamethoxazole $(\rho=0.67)$, streptomycin and sulfisoxazole $(\rho=0.51)$, streptomycin and tetracycline $(\rho=0.49)$, gentamicin and sulfisoxazole $(\rho=0.49)$, and ampicillin and streptomycin $(\rho=0.46)$ (Table 3$)$. All pairwise relationships were positive.

\section{Differences in AMR of fecal $E$. coli isolates between poultry species}

The odds of resistance to tetracycline (odds ratio $=5.89$, 95\% CI $=1.71-20.29, P=0.005$ ) and ampicillin (odds ratio $=4.06,95 \% \mathrm{CI}=1.24-13.25, P=0.020)$ were significantly higher in turkey $E$. coli isolates compared to isolates from all the other poultry species. The rate of MDR was significantly higher (incidence rate ratio $=1.99,95 \% \mathrm{CI}=$ $1.16-3.40, P=0.012$ ) in turkey $E$. coli isolates compared to isolates from all the other poultry species.

\section{Discussion}

Our study evaluated AMR in fecal E. coli and Salmonella enterica isolates from birds from small flocks experiencing morbidity, mortality, or production issues, and found a relatively high frequency of resistance to antimicrobials commonly used to treat bacterial infections in poultry. Differences in flock characteristics, including 
Table 2 Most common antimicrobial resistance patterns of fecal Escherichia coli isolates from Ontario small poultry flocks, by poultry species

\begin{tabular}{|c|c|c|c|}
\hline Poultry species & Antimicrobial resistance pattern ${ }^{\mathrm{A}}$ & Number of antimicrobial classes in pattern (multidrug resistant) ${ }^{B}$ & $n(\%)^{C}$ \\
\hline \multirow[t]{6}{*}{ Chicken $(N=358)$} & STR-TET & 2 (no) & $19(5.31)$ \\
\hline & AMP-STR-TET & 3 (yes) & $22(6.15)$ \\
\hline & SSS-STR-TET & 3 (yes) & $6(1.68)$ \\
\hline & GEN-SSS-STR & 2 (no) & $6(1.68)$ \\
\hline & GEN-SSS-STR-TET & 3 (yes) & $5(1.40)$ \\
\hline & AMP-SSS-STR-STX-TET & 4 (yes) & $5(1.40)$ \\
\hline \multirow[t]{5}{*}{ Turkey $(N=27)$} & AMP-TET & 2 (no) & $3(11.11)$ \\
\hline & STR-TET & 2 (no) & $2(7.41)$ \\
\hline & SSS-STR-TET & 3 (yes) & $2(7.41)$ \\
\hline & AMP-SSS-STR-STX-TET & 4 (yes) & $2(7.41)$ \\
\hline & AMP-CHL-CIP-NAL-SSS-STR-TET & 6 (yes) & $2(7.41)$ \\
\hline $\operatorname{Duck}(N=24)$ & SSS-STX-TET & 2 (no) & $2(8.33)$ \\
\hline \multirow[t]{2}{*}{ Game bird $(N=24)$} & STR-TET & 2 (no) & $6(25.00)$ \\
\hline & SSS-STR-TET & 3 (yes) & $2(8.33)$ \\
\hline
\end{tabular}

${ }^{A}$ Resistance to 14 selected antimicrobials (including amoxicillin-clavulanic acid, ceftriaxone, cefoxitin, meropenem, azithromycin), as determined by a broth microdilution technique. GEN gentamicin, STR streptomycin, AMP ampicillin, SSS sulfisoxazole, STX trimethoprim-sulfamethoxazole, CHL chloramphenicol, CIP ciprofloxacin, NAL nalidixic acid, TET tetracycline

${ }^{B}$ An isolate was defined as multidrug resistant if it was non-susceptible to at least one antimicrobial in $\geq 3$ antimicrobial classes (Aminoglycosides: GEN, STR; $\beta$ Lactams: AMP; Folate biosysnthesis pathway inhibitors: SSS, STX; Phenicols: CHL; Quinolones: CIP, NAL; Tetracyclines: TET)

${ }^{C}$ Number and percentage of isolates with each antimicrobial resistance pattern. For chicken, only patterns with $\geq 5$ isolates are shown, and for other poultry species, only patterns with $\geq 2$ isolates are shown

poultry species, health status (diseased or healthy), or husbandry (commercial or non-commercial), or dissimilarities in study design, analytical methods (isolate-level or flock-level analysis), sampling protocols (on-farm, at slaughter plants, or at diagnostic laboratories), or antimicrobial susceptibility testing (disk diffusion or broth microdilution) can make comparisons to other studies difficult. Our comparisons are limited to results from studies that evaluated AMR in fecal E. coli and Salmonella spp. in small flocks using samples collected on-farm, at diagnostic laboratories, or at slaughter.

Outbreaks of human salmonellosis linked to contact with small flocks have been reported in the United States [4, 28], Bangladesh [29], and Chile [30]. The Salmonella serotypes identified in our study (Anatum, Indiana, Ouakam, Uganda, and Montevideo) are not among the most prevalent commercial poultry-associated serotypes (Kentucky, Enteritidis, Heidelberg, and Typhimurium) in Canada [31] or the United States [32], and they are less frequently associated with human salmonellosis cases in Canada [33]. Nonetheless, the presence of AMR in Salmonella in small flocks is concerning because of the risk that resistant salmonellae pose in human cases with bacteremia or a compromised immune system. Although the frequency of Salmonella enterica was very low in our study [26], and many of the Salmonella isolates were pan-susceptible, some were resistant to streptomycin alone, or to streptomycin, sulfisoxazole, trimethoprim-sulfamethoxazole, and tetracycline. Our findings agree with a recent study that evaluated AMR in Salmonella isolated from small flock cases at the California Animal Health and Food Safety Laboratory System, and found resistance to streptomycin, sulfonamides, and tetracycline [34].

In the fecal E. coli isolates in our study, there was a very low frequency of resistance to cephalosporins, carbapenems, macrolides, and quinolones, which are antimicrobials classified in Canada as being of very high or high importance in human medicine [20]. This is an encouraging finding from a human health viewpoint because flock owners can be exposed to antimicrobial resistant zoonotic pathogens $[6,13,15]$ through direct contact with their birds [10] or their environment [11], or consumption of contaminated meat or eggs $[8,9]$. However, there was a moderate to high frequency of resistance to tetracycline, streptomycin, sulfonamides, and ampicillin; antimicrobials frequently used to treat bacterial infections in poultry [35]. These findings were in accordance with other small flock studies in Canada (tetracycline resistance $37 \%$, streptomycin $21 \%$, sulfisoxazole 16\%, ampicillin 15\%) [25] and Ecuador (tetracycline $69 \%$, streptomycin $42 \%$, sulfisoxazole $65 \%$, ampicillin $45 \%$ ) [36], and one study of commercial broiler chicken flocks in Canada (tetracycline 53\%, streptomycin 33\%, sulfisoxazole $18 \%$, ampicillin 38\%) [23].

Our cluster and correlation analyses of the chicken E. coli isolates showed that there was concurrent resistance to 


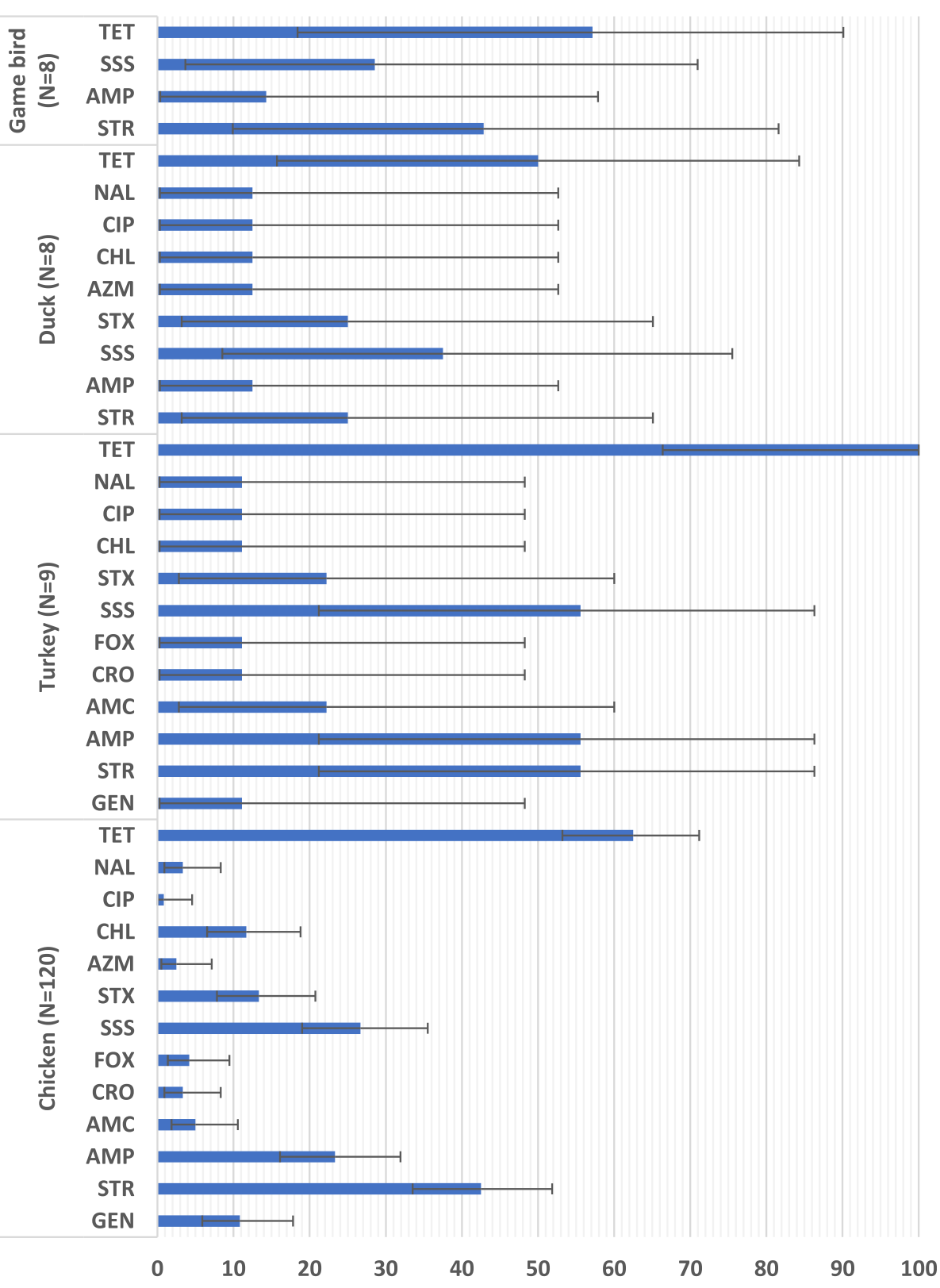

Percentage of E. coli-positive samples

Fig. 1 Percentage of Escherichia coli-positive fecal samples from Ontario small poultry flocks with production, clinical illness, or mortality issues that were resistant to antimicrobials, by poultry species. ${ }^{A B C D}$. ASample-level resistance to 14 selected antimicrobials (including meropenem), as determined by a broth microdilution technique. $\mathrm{GEN}=$ gentamicin; $\mathrm{STR}=$ streptomycin; $\mathrm{AMP}=$ ampicillin; $\mathrm{AMC}=$ amoxicillin-clavulanic acid; $\mathrm{CRO}=$ ceftriaxone; FOX = cefoxitin; SSS = sulfisoxazole; STX = trimethoprim-sulfamethoxazole; $\mathrm{AZM}=$ azithromycin; $\mathrm{CHL}=$ chloramphenicol; $\mathrm{CIP}=$ ciprofloxacin; $\mathrm{NAL}=$ nalidixic acid; TET $=$ tetracycline. Only antimicrobials for which resistance was detected are shown. ${ }^{\mathrm{B}}$ Antimicrobial classes. Aminoglycosides (GEN, STR); $\beta$-Lactams (AMP, AMC, CRO, FOX); Folate biosynthesis pathway inhibitors (SSS, STX); Macrolides (AZM); Phenicols (CHL); Quinolones (CIP, NAL); Tetracyclines (TET). ${ }^{C}$ For each poultry species, estimates were computed by dividing the number of samples resistant to an antimicrobial by the total number of E. coli-positive samples. A sample was considered to be resistant to a specific antimicrobial if at least one isolate from a pooled cecal sample was resistant. ${ }^{D}$ Exact binomial $95 \%$ confidence interval for the proportion of antimicrobial resistant samples

streptomycin, tetracycline, sulfisoxazole, and trimethoprimsulfamethoxazole; clusters of turkey, duck, and game bird isolates included many of the same antimicrobials. Our cluster analyses also showed that there was concurrent resistance to cefoxitin and ceftriaxone in the chicken E. coli isolates and to amoxicillin-clavulanic acid, cefoxitin, and 
a) Chicken ( $\mathrm{N}=358)$

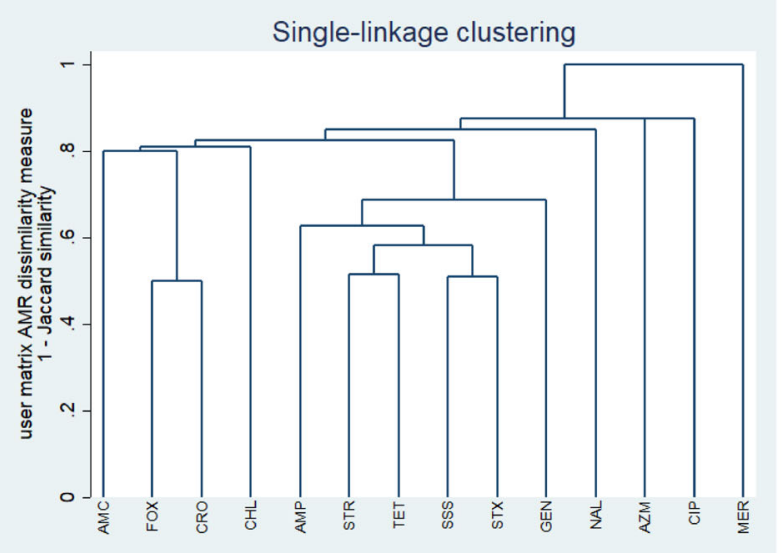

c) Duck ( $N=24)$

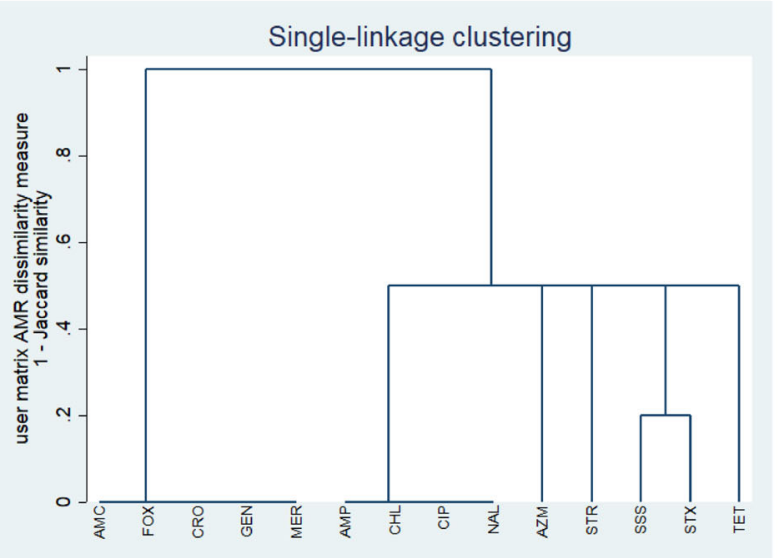

b) Turkey ( $\mathrm{N}=27)$

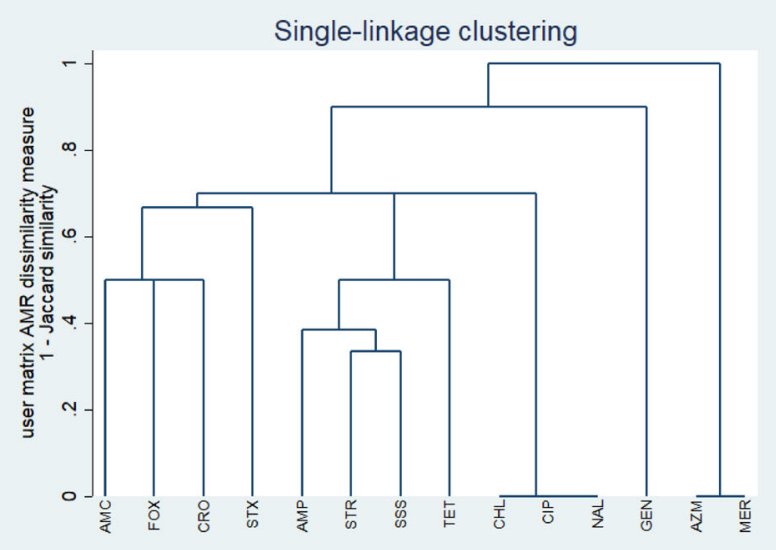

d) Game bird (N=24)

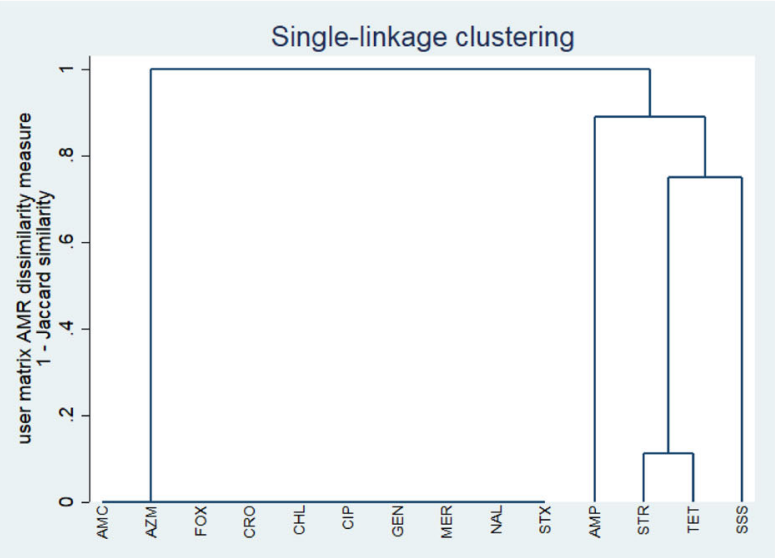

Fig. 2 Single-linkage clustering dendrograms of resistance of fecal Escherichia coli isolates to antimicrobials, by poultry species ${ }^{A B C}$. ${ }^{A} G E N=$ gentamicin; STR = streptomycin; AMP = ampicillin; $A M C=$ amoxicillin-clavulanic acid; $C R O=$ ceftriaxone; FOX = cefoxitin; MER = meropenem; SSS = sulfisoxazole; STX = trimethoprim-sulfamethoxazole; $\mathrm{AZM}=$ azithromycin; $\mathrm{CHL}=$ chloramphenicol; $\mathrm{CIP}=$ ciprofloxacin; $\mathrm{NAL}=$ nalidixic acid; TET = tetracycline. ${ }^{B}$ A cluster analysis, using the Jaccard binary similarity coefficient, was used to compare individual antimicrobials with respect to their similarity in the resistance status of E. coli. The Jaccard distance measures dissimilarity between antimicrobials and is obtained by subtracting the Jaccard binary similarity coefficient from one [27]. A high dissimilarity measure indicates that relatively few isolates were resistant to both antimicrobials, a low dissimilarity measure indicates that a relatively high proportion of isolates were resistant to both antimicrobials, and a dissimilarity measure of zero indicates that all isolates were susceptible to both antimicrobials

ceftriaxone in the turkey $E$. coli isolates. The moderate frequency of MDR in the turkey and chicken $E$. coli isolates (and to a lesser extent in the duck and game bird E. coli isolates), and the strong correlations in resistance of the chicken $E$. coli isolates to antimicrobials commonly used to treat bacterial infections of poultry, highlight the importance of judicious antimicrobial use to limit the development and dissemination of multidrug resistant bacteria in small flocks $[12,13]$.

Our regression models showed that there were higher probabilities of resistance to tetracycline and ampicillin in the E. coli isolates of turkeys when compared to isolates obtained from all the other poultry species. Moreover, the rate of MDR was significantly higher in the turkey isolates compared to all the other species.
Differences in AMR between poultry species might be explained by variation in antimicrobial use or husbandry practices. However, these findings should be interpreted cautiously because chicken isolates were over-represented in our study. Therefore, further studies are needed to assess factors that might have a role in the development of AMR in commensal and pathogenic enteric bacteria of small flocks.

Limitations of this study include a sampling bias, as most submissions came from southwestern and eastern Ontario, which might have been the consequence of the geographic proximity to the diagnostic laboratories in Guelph and Kemptville, respectively [26]. Also, small flocks were not randomly selected, and our study included only owners who had a flock veterinarian, as 
Table 3 Pairwise correlations between antimicrobials (with respect to resistance) of fecal Escherichia coli isolates of chickens from Ontario small poultry flocks $(n=358)^{\mathrm{AB}}$

\begin{tabular}{|c|c|c|c|c|c|c|c|c|c|c|c|c|c|}
\hline & AMC & AMP & AZM & FOX & CRO & $\mathrm{CHL}$ & CIP & GEN & NAL & SSS & STR & STX & TET \\
\hline AMC & 1.000 & & & & & & & & & & & & \\
\hline AMP & 0.347 & 1.000 & & & & & & & & & & & \\
\hline AZM & - & - & 1.000 & & & & & & & & & & \\
\hline FOX & 0.304 & - & 0.189 & 1.000 & & & & & & & & & \\
\hline CRO & 0.344 & 0.244 & 0.214 & 0.667 & 1.000 & & & & & & & & \\
\hline $\mathrm{CHL}$ & 0.322 & 0.262 & 0.197 & 0.197 & & 1.000 & & & & & & & \\
\hline CIP & - & - & - & - & - & 0.237 & 1.000 & & & & & & \\
\hline GEN & 0.192 & - & - & - & 0.189 & - & - & 1.000 & & & & & \\
\hline NAL & - & - & - & - & - & - & 0.350 & & 1.000 & & & & \\
\hline SSS & 0.283 & 0.310 & - & - & - & 0.283 & - & 0.487 & 0.183 & 1.000 & & & \\
\hline STR & 0.193 & 0.458 & - & - & - & - & - & 0.332 & 0.235 & 0.508 & 1.000 & & \\
\hline STX & 0.295 & 0.364 & 0.223 & - & - & 0.264 & - & - & 0.295 & 0.667 & 0.315 & 1.000 & \\
\hline TET & - & 0.329 & - & - & - & - & - & - & - & 0.294 & 0.490 & 0.204 & 1.000 \\
\hline
\end{tabular}

${ }^{A}$ GEN gentamicin, STR streptomycin, AMP ampicillin, AMC amoxicillin-clavulanic acid, CRO ceftriaxone, FOX cefoxitin, MER meropenem, SSS sulfisoxazole, STX trimethoprim-sulfamethoxazole, AZM azithromycin, CHL chloramphenicol, CIP ciprofloxacin, NAL nalidixic acid, TET tetracycline

${ }^{B}$ Spearman rank correlation, with a Bonferroni correction $(\alpha / 14)$ to adjust for multiple comparisons, was used to measure the strength and direction of the relationships between individual antimicrobials with respect to resistance of $E$. coli at the isolate-level (chicken isolates only). Only statistically significant $(P \leq$ 0.0036) correlations are shown

this is a laboratory submission requirement. We also used fecal samples from diagnostic submissions and not from healthy birds. Our study might therefore overestimate the frequency of AMR because samples came from birds that might have already been treated with antimicrobials.

\section{Conclusions}

Our study enhances the knowledge on AMR of small flocks by evaluating the AMR patterns of E. coli and Salmonella isolates from chickens, turkeys, ducks, and game birds. These results can be used as a benchmark for ongoing monitoring of AMR in enteric bacteria of small flocks in Ontario, in light of the recently amended antimicrobial use regulation in Canada. Ultimately, the findings derived from this study can be used to educate veterinarians and small flock owners about issues surrounding AMR, with a goal of reducing the presence of multidrug resistant bacteria in small flocks and mitigating the risk they might pose to public health.

\section{Methods}

\section{Study design}

Samples were obtained through a prospective surveillance study of small flocks conducted in Ontario between October 2015 and September 2017, which is described in detail elsewhere [26]. In brief, a small flock was defined as a non-commercial poultry flock composed of not more than 299 broiler chickens, 99 layer chickens, 49 turkeys, 300 waterfowl, or 300 game birds. Small flock owners who had issues with production, clinical illness, or mortality in their flock were provided the opportunity to submit birds for diagnostic testing for a discounted fee. Submissions $(n=160)$ were made to the Animal Health Laboratory, University of Guelph through the owner's veterinarian. A maximum of 5 sick and/or dead birds of one species from the same flock constituted a submission. Live birds submitted to the lab were euthanized using carbon dioxide.

\section{Sample collection and bacterial isolation}

All bacterial isolation and antimicrobial susceptibility testing were conducted at the Animal Health Laboratory, Guelph, Ontario. From each submission, one pooled cecal sample was collected (from all of the birds of the same species from the same flock in the submission) and tested for fecal E. coli and Salmonella spp. Cecal material was directly plated on MacConkey and Hektoen enteric agars (Oxoid Ltd., Nepean, ON) for E. coli isolation, and inoculated into buffered peptone water (Bio-Media Unlimited Ltd., Toronto, ON) for Salmonella spp. preenrichment. Aliquots of buffered peptone water were then transferred to Hajna tetrathionate (Animal Health Laboratory, Guelph, ON) and Rappaport Vasiliadis broths (BioMedia Unlimited Ltd.) for Salmonella spp. enrichment, followed by plating on brilliant green (Bio-Media Unlimited Ltd.) and XLT-4 agars (Oxoid Ltd.). Presumptive E. coli and Salmonella spp. colonies were identified using matrix-assisted laser desorption ionization time-of-flight mass spectrometry (Bruker Ltd., Billerica, MA) [37]. Salmonella-positive isolates were submitted to the OIE (World Organisation for Animal Health) Salmonella 
reference laboratory at the National Microbiology Laboratory in Guelph for serotyping according to published methods [38].

\section{Antimicrobial susceptibility testing and classification}

Three different isolates from each $E$. coli-positive sample and one isolate from each Salmonella-positive sample were purposively selected. Susceptibility testing of $E$. coli and Salmonella isolates to 14 antimicrobials was conducted using automated broth microdilution (Sensititre; Trek Diagnostic Systems Inc., Westlake, $\mathrm{OH}$ ) with the National Antimicrobial Monitoring System CMV4AGNF panel [22]. Based on the interpretive standards of the Canadian Integrated Program for Antimicrobial Resistance Surveillance [22], E. coli and Salmonella isolates with a minimum inhibitory concentration lower than or equal to the Susceptible breakpoint were classified as susceptible, whereas those with a minimum inhibitory concentration higher than the Susceptible breakpoint were considered to be resistant. The Susceptible breakpoints are: amoxicillin-clavulanic acid $(\leq 8 / 4 \mu \mathrm{g} / \mathrm{mL})$; ampicillin $(\leq 8 \mu \mathrm{g} / \mathrm{mL})$; azithromycin $(\leq 16 \mu \mathrm{g} / \mathrm{mL})$; cefoxitin $(\leq 8 \mu \mathrm{g} / \mathrm{mL})$; ceftriaxone $(\leq 1 \mu \mathrm{g} / \mathrm{mL})$; chloramphenicol $(\leq 8 \mu \mathrm{g} / \mathrm{mL})$; ciprofloxacin $(\leq 0.06 \mu \mathrm{g} / \mathrm{mL})$; gentamicin $(\leq$ $4 \mu \mathrm{g} / \mathrm{mL})$; meropenem $(\leq 1 \mu \mathrm{g} / \mathrm{mL})$; nalidixic acid $(\leq$ $16 \mu \mathrm{g} / \mathrm{mL}) ;$ streptomycin $(\leq 16 \mu \mathrm{g} / \mathrm{mL})$; sulfisoxazole $(\leq$ $256 \mu \mathrm{g} / \mathrm{mL})$; tetracycline $(\leq 4 \mu \mathrm{g} / \mathrm{mL})$; and trimethoprimsulfamethoxazole $(\leq 2 / 38 \mu \mathrm{g} / \mathrm{mL})[22]$.

An isolate was defined as multidrug resistant if it was non-susceptible to at least one antimicrobial in $\geq 3$ different antimicrobial classes [39]. In our study, classes included: Aminoglycosides (gentamicin, streptomycin); $\beta$ Lactams (amoxicillin-clavulanic acid, ampicillin, cefoxitin, ceftriaxone, meropenem); Folate biosynthesis pathway inhibitors (sulfisoxazole, trimethoprim-sulfamethoxazole); Macrolides (azithromycin); Phenicols (chloramphenicol); Quinolones (ciprofloxacin, nalidixic acid); and Tetracyclines (tetracycline).

\section{Data analysis}

Antimicrobial susceptibility data were entered into a spreadsheet (Microsoft Excel 2016, Microsoft Corporation, Redmond, WA), reviewed for missing values, and subsequently transferred into a statistical software program (STATA Intercooled, version 14.2, Stata Corporation, College Station, TX) for analysis.

For each poultry species (chicken, turkey, duck, and game bird), estimates of the proportion of E. coli and Salmonella isolates that were resistant to each of the 14 tested antimicrobials were computed by dividing the number of isolates resistant to an antimicrobial by the total number of isolates tested for the antimicrobial. In addition, estimates of the proportion of isolates that showed multidrug resistance (MDR) were computed by dividing the number of multidrug resistant isolates by the total number of isolates tested.

Further, for each poultry species, estimates of the percentage of $E$. coli-positive samples that were resistant to each of the 14 tested antimicrobials were computed by dividing the number of samples resistant to an antimicrobial by the total number of $E$. coli-positive samples. A sample was considered to be resistant to a specific antimicrobial if at least one isolate from a pooled cecal sample was resistant. For all estimates, exact binomial 95\% confidence intervals (CIs) were calculated.

To compare individual antimicrobials with respect to their similarity in the resistance status of $E$. coli, a cluster analysis, using the Jaccard binary similarity coefficient, was performed for each poultry species. The number of isolates that are resistant to both antimicrobials and the number that are resistant to one yet susceptible to the other are used in the calculation of the coefficient. Dendrograms were constructed using the single-linkage clustering method with the Jaccard distance. The Jaccard distance measures dissimilarity between antimicrobials and is obtained by subtracting the Jaccard binary similarity coefficient from one [27]. Thus, a high dissimilarity measure indicates that relatively few isolates were resistant to both antimicrobials, whereas a low dissimilarity measure indicates that a relatively high proportion of isolates were resistant to both antimicrobials. A dissimilarity measure of zero indicates that all isolates were susceptible to both antimicrobials.

Further, to measure the strength and direction of the relationships between individual antimicrobials with respect to resistance of E. coli at the isolate-level, Spearman's rank correlation coefficients were calculated; only chicken isolates were evaluated. A Bonferroni correction was used to adjust for multiple comparisons among antimicrobials, with $P \leq 0.0036$ ( $\alpha$ of $0.05 / 14$ ) indicating a significant correlation.

To identify differences in E. coli resistance between poultry species, logistic regression was used; only antimicrobials for which $\geq 5 \%$ of the isolates were resistant were evaluated. Therefore, 6 of 14 antimicrobials were analyzed: ampicillin, gentamicin, streptomycin, sulfisoxazole, trimethoprim-sulfamethoxazole, and tetracycline. Four population-averaged models were built for each antimicrobial using the generalized estimating equation method, with a robust variance estimate and exchangeable correlation structure to account for sample-level clustering. In these univariable models, the binary (yes/ no) dependent variable represented the frequency of resistance to the antimicrobial, while the independent variable was poultry species (binary variable: chickens compared to all the other poultry species; turkeys compared to all the other poultry species; ducks compared to all the other poultry species; and game birds 
compared to all the other poultry species). This method of grouping species together was preferred over analyzing species as a categorical variable because of the limited number of isolates from turkeys, ducks, and game birds. A $P$-value $\leq 0.05$ on the Wald $\chi^{2}$ test indicated a statistically significant association.

In addition, four Poisson regression models were built to identify differences in E. coli MDR between poultry species using the generalized estimating equation method described above. The dependent variable was the number of antimicrobial classes to which an isolate was resistant; as seven antimicrobial classes were studied, this count potentially ranged from zero to seven. The independent variable was the poultry species (binary variable; described above).

\section{Abbreviations}

AMR: Antimicrobial resistance; Cl: Confidence interval; MDR: Multidrug resistance

\section{Acknowledgements}

The authors thank all the veterinarians and small flock owners who submitted birds and participated in this study. We also thank the laboratory staff of the Animal Health Laboratory for coordinating and processing all submissions and performing AMR testing.

\section{Authors' contributions}

CV, MTG, MLB, and LS designed the study. CV analyzed the data, interpreted results, wrote the first draft of the manuscript, responded to editorial comments, and prepared the final manuscript for submission. MTG provided advice on data analysis and contributed to addressing editorial comments and preparing the final manuscript for submission. MLB coordinated the sample collection. DS coordinated the laboratory testing (bacterial isolation and antimicrobial susceptibility testing). MTG, MLB, DS, PB, and LS provided advice on interpretation of results, reviewed and commented on manuscript drafts. All authors read and approved the final manuscript.

\section{Funding}

The surveillance project was funded by the OMAFRA-University of Guelph Strategic Partnership (grant UofG 2015-2282), under the Disease Surveillance Plan, which was a joint federal-provincial Growing Forward 2 project. The antimicrobial susceptibility testing was funded by the Ontario Agri-Food Innovation Alliance, under the Disease Surveillance Plan (Project \# 009098). The funders had no role in the design of the study, data collection, analyses, interpretation of data, decision to publish, or preparation of the manuscript.

\section{Availability of data and materials}

The datasets generated and/or analyzed during the current study are not publicly available, as the data will be used for additional epidemiological analyses.

\section{Ethics approval and consent to participate}

This study was approved by the University of Guelph Research Ethics Board (REB-16-12-657). In accordance with the REB, small flock owners were required to read and sign a written Consent to Participate in Research form at the time of submission of birds for diagnostic testing.

\section{Consent for publication}

Not applicable.

\section{Competing interests}

The authors declare that they have no competing interests.

\section{Author details}

'Department of Pathobiology, College of Veterinary Medicine, University of Illinois at Urbana-Champaign, Urbana, Illinois 61802, USA. ${ }^{2}$ Department of Population Medicine, Ontario Veterinary College, University of Guelph,
Guelph, Ontario N1G 2W1, Canada. ${ }^{3}$ Animal Health Laboratory, University of Guelph, Guelph, Ontario N1G 2W1, Canada. ${ }^{4}$ Department of Pathobiology, Ontario Veterinary College, University of Guelph, Guelph, Ontario N1G 2W1, Canada.

Received: 21 February 2019 Accepted: 25 November 2019

Published online: 21 December 2019

\section{References}

1. Burns TE, Kelton D, Ribble C, Stephen C. Preliminary investigation of bird and human movements and disease-management practices in noncommercial poultry flocks in southwestern British Columbia. Avian Dis. 2011;55(3):350-7.

2. Elkhoraibi C, Blatchford RA, Pitesky ME, Mench JA. Backyard chickens in the United States: a survey of flock owners. Poult Sci. 2014;93:2920-31.

3. Mainali C, Houston I. Small poultry flocks in Alberta: demographics and practices. Avian Dis. 2017;61(1):46-54.

4. Behravesh CB, Brinson D, Hopkins BA, Gomez TM. Backyard poultry flocks and salmonellosis: a recurring, yet preventable public health challenge. Clin Infect Dis. 2014;58(10):1432-8.

5. Grunkemeyer VL. Zoonoses, public health, and the backyard poultry flock. Vet Clin Exot Anim. 2011;14:477-90.

6. Pohjola L, Nykäsenoja S, Kivistö R, Soveri T, Huovilainen A, Hänninen ML, Fredriksson-Ahomaa M. Zoonotic public health hazards in backyard chickens. Zoonoses Public Health. 2016;63(5):420-30.

7. Whitfield Y, Johnson K, Hobbs L, Middleton D, Dhar B, Vrbova L. Descriptive study of enteric zoonoses in Ontario, Canada, from 2010 - 2012. BMC Public Health. 2017:17(1):217.

8. Middleton D, Savage R, Tighe MK, Vrbova L, Walton R, Whitfield Y, Varga C, Lee B, Rosella L, Dhar B, Johnson C, Ahmed R, Allen VG, Crowcroft NS. Risk factors for sporadic domestically acquired Salmonella serovar Enteritidis infections: a case-control study in Ontario, Canada, 2011. Epidemiol Infect. 2014;142(7):1411-21

9. Varga C, Middleton D, Walton R, Savage R, Tighe MK, Allen V, Ahmed R, Rosella L. Evaluating risk factors for endemic human Salmonella Enteritidis infections with different phage types in Ontario, Canada using multinomial logistic regression and a case-case study approach. BMC Public Health. 2012;12:866.

10. Basler C, Nguyen TA, Anderson TC, Hancock T, Behravesh CB. Outbreaks of human Salmonella infections associated with live poultry, United States, 1990-2014. Emerg Infect Dis. 2016;22(10):1705-11.

11. Finley RL, Collignon P, Larsson DGJ, McEwen SA, Li X-Z, Gaze WH, Topp E. The scourge of antibiotic resistance: the important role of the environment. Clin Infect Dis. 2013;57(5):704-10.

12. Levy SB, Marshall B. Antibacterial resistance worldwide: causes, challenges and responses. Nat Med. 2004;10(Suppl):122-9. https:/doi.org/10.1038/nm1145.

13. Nhung NT, Chansiripornchai N, Carrique-Mas JJ. Antimicrobial resistance in bacterial poultry pathogens: A review. Front Vet Sci. 2017;4:126.

14. World Health Organization. 2018. Antimicrobial resistance: World Health Organization. http://www.who.int/mediacentre/factsheets/fs194/en/. Accessed 10 Jan 2019

15. Varga C, Guerin MT, Brash ML, Slavic D, Boerlin P, Susta L. Antimicrobial resistance in campylobacter jejuni and campylobacter coli isolated from small poultry flocks in Ontario, Canada: a two-year surveillance study. PLoS One. 2019;14(8):e0221429. https://doi.org/10.1371/journal.pone.0221429.

16. Caniça M, Manageiro V, Abriouel H, Moran-Gilad J, Franz CMAP. Antibiotic resistance in foodborne bacteria. Trends Food Sci Technol. 2018. https://doi.org/10.1016/j.tifs.2018.08.001.

17. Helke KL, McCrackin MA, Galloway AM, Poole AZ, Salgado CD, Marriott BP. Effects of antimicrobial use in agricultural animals on drug-resistant foodborne salmonellosis in humans: a systematic literature review. Crit Rev Food Sci Nutr. 2017;57(3):472-88.

18. Callens B, Cargnel M, Sarrazin S, Dewulf J, Hoet B, Vermeersch K, Wattiau P, Welby $S$. Associations between a decreased veterinary antimicrobial use and resistance in commensal Escherichia coli from Belgian livestock species (2011-2015). Prev Vet Med. 2018;157:50-8.

19. Chalmers G, Cormier AC, Nadeau M, Côté G, Reid-Smith RJ, Boerlin P. Determinants of virulence and of resistance to ceftiofur, gentamicin, and spectinomycin in clinical Escherichia coli from broiler chickens in Québec. Canada Vet Microbiol. 2017;203:149-57. 
20. Government of Canada. Health Canada. 2009. Categorization of Antimicrobial Drugs Based on Importance in Human Medicine. https://www.canada.ca/en/health-canada/services/drugs-healthproducts/veterinary-drugs/antimicrobial-resistance/categorizationantimicrobial-drugs-based-importance-human-medicine.html. Accessed 20 Dec 2018.

21. Government of Canada. Food and Drugs Act. Regulations Amending the Food and Drug Regulations (Veterinary Drugs-Antimicrobial Resistance). 2017; Vol. 151, No. 10. http://gazette.gc.ca/rp-pr/p2/2017/2017-05-17/html/ sor-dors76-eng.html. Accessed 5 Dec 2018.

22. Government of Canada. Canadian Integrated Program for Antimicrobial Resistance Surveillance (CIPARS) 2016 Annual Report. In: Chapter 5 Design and methods. http://publications.gc.ca/collections/collection_2018/aspcphac/HP2-4-2016-eng.pdf. Accessed 5 Jan 2019.

23. Agunos A, Arsenault RK, Avery BP, Deckert AE, Gow SP, Janecko N, Léger DF, Parmley EJ, Reid-Smith RJ, McEwen SA. Changes in antimicrobial resistance levels among Escherichia coli, Salmonella, and Campylobacter in Ontario broiler chickens between 2003 and 2015. Can J Vet Res. 2018;82(3): 163-77.

24. Varga C, Brash ML, Slavic D, Boerlin P, Ouckama R, Weisz A, Petrik M, Philippe C, Barham M, Guerin MT. Evaluating virulence-associated genes and antimicrobial resistance of avian pathogenic Escherichia coli isolates from broiler and broiler breeder chickens in Ontario, Canada. Avian Dis. 2018; 62(3):291-9.

25. Lebert L, Martz S-L, Janecko N, Deckert AE, Agunos A, Reid A, SA ME. Prevalence and antimicrobial resistance among Escherichia coli and Salmonella in Ontario smallholder chicken flocks. Zoonoses Public Health. 2017. https://doi.org/10.1111/zph.12381.

26. Brochu NM, Guerin MT, Varga C, Lillie BN, Brash ML, Susta L. A two-year prospective study of small poultry flocks in Ontario, Canada: part 1, prevalence of viral and bacterial pathogens. J Vet Diagn Investig. 2019;31(3): 327-35. https://doi.org/10.1177/1040638719843577.

27. Kaufman L, Rousseeuw PJ. Finding groups in data: an introduction to cluster analysis. New York: Wiley; 1990.

28. Anderson TC, Nguyen TA, Adams JK, Garrett NM, Bopp CA, Baker JB, McNeil C, Torres P, Ettestad PJ, Erdman MM, Brinson DL, Gomez TM, Barton BC. Multistate outbreak of human Salmonella Typhimurium infections linked to live poultry from agricultural feed stores and mail-order hatcheries, United States 2013. One Health. 2016;2:144-9.

29. Shanta IS, Hasnat MA, Zeidner N, Gurley ES, Azziz-Baumgartner E, Sharker MAY, Hossain K, Khan SU, Haider N, Bhuyan AA, Hossain MA, Luby SP. Raising backyard poultry in rural Bangladesh: financial and nutritional benefits, but persistent risky practices. Transbound Emerg Dis. 2017;64(5): 1454-64.

30. Toro M, Rivera D, Toledo V, Campos-Vargas R, Allard MW, Hamilton-West C, Moreno-Switt Al. Genomics of Salmonella contaminating backyard production systems reveals persistence and transmission of genetically related Salmonella on a farm basis. Zoonoses Public Health. 2018;65(8): $1008-14$.

31. Sivaramalingam T, McEwen SA, Pearl DL, Ojkic D, Guerin MT. A temporal study of Salmonella serovars from environmental samples from poultry breeder flocks in Ontario between 1998 and 2008. Can J Vet Res. 2013;77(1): $1-11$.

32. Shah DH, Paul NC, Sischo WC, Crespo R, Guard J. Population dynamics and antimicrobial resistance of the most prevalent poultry-associated Salmonella serotypes. Poult Sci. 2017;96(3):687-702.

33. Parmley EJ, Pintar K, Majowicz S, Avery B, Cook A, Jokinen C, Gannon V, Lapen DR, Topp E, Edge TA, Gilmour M, Pollari F, Reid-Smith R, Irwin R. A Canadian application of one health: integration of Salmonella data from various Canadian surveillance programs (2005-2010). Foodborne Pathog Dis. 2013;10(9):747-56.

34. Clothier KA, Kim P, Mete A, Hill AE. Frequency, serotype distribution, and antimicrobial susceptibility patterns of Salmonella in small poultry flocks in California. J Vet Diagn Investig. 2018;30(3):471-5.

35. Agunos A, Léger D, Carson C. Review of antimicrobial therapy of selected bacterial diseases in broiler chickens in Canada. Can Vet J. 2012;53:1289-300.

36. Moser KA, Zhang L, Spicknall I, Braykov NP, Levy K, Marrs CF, Foxman B, Trueba G, Cevallos W, Goldstick J, Trostle J, Eisenberg JNS. The role of mobile genetic elements in the spread of antimicrobial-resistant Escherichia coli from chickens to humans in small-scale production poultry operations in rural Ecuador. Am J Epidemiol. 2018;187(3):558-67.
37. Dingle TC, Butler-Wu SM. Maldi-tof mass spectrometry for microorganism identification. Clin Lab Med. 2013;33(3):589-609. https://doi.org/10.1016/j.cll. 2013.03.001.

38. Grimont PAD, Weill F-X. Antigenic formulae of the Salmonella serovars. In: World Health Organization Collaborating Centre for Reference and Research on Salmonella. 9th ed. Paris: Pasteur Institute; 2007. https://www.pasteur.fr/ sites/default/files/veng_0.pdf. Accessed 15 Jun 2019

39. Magiorakos AP, Srinivasan A, Carey RB, Carmeli Y, Falagas ME, Giske CG, Harbarth S, Hindler JF, Kahlmeter G, Olsson-Liljequist B, Paterson DL, RiceLB SJ, Struelens MJ, Vatopoulos A, Weber JT, Monnet DL. Multidrug-resistant, extensively drug-resistant and pandrug-resistant bacteria: an international expert proposal for interim standard definitions for acquired resistance. Clin Microbiol Infect. 2012;18:268-81.

\section{Publisher's Note}

Springer Nature remains neutral with regard to jurisdictional claims in published maps and institutional affiliations.
Ready to submit your research? Choose BMC and benefit from:

- fast, convenient online submission

- thorough peer review by experienced researchers in your field

- rapid publication on acceptance

- support for research data, including large and complex data types

- gold Open Access which fosters wider collaboration and increased citations

- maximum visibility for your research: over $100 \mathrm{M}$ website views per year

At BMC, research is always in progress.

Learn more biomedcentral.com/submissions 